\title{
A propos de l'APAD et des sciences économiques
}

\section{Pierre-Yves Le Meur}

\section{(2) OpenEdition}

Journals

Édition électronique

URL : http://journals.openedition.org/apad/3523

DOI : 10.4000/apad.3523

ISSN : 1950-6929

Éditeur

LIT Verlag

Édition imprimée

Date de publication : 1 juin 1993

\section{Référence électronique}

Pierre-Yves Le Meur, «A propos de l'APAD et des sciences économiques », Bulletin de l'APAD [En ligne], 5| 1993, mis en ligne le 04 juin 2008, consulté le 08 septembre 2020. URL : http:// journals.openedition.org/apad/3523; DOI : https://doi.org/10.4000/apad.3523

Ce document a été généré automatiquement le 8 septembre 2020

Bulletin de l'APAD 


\title{
A propos de l'APAD et des sciences économiques
}

\author{
Pierre-Yves Le Meur
}

1 Cette petite lettre a pour objet une question que je me pose, au fil de mes lectures assidues des différents bulletins de l'APAD, et en particulier des textes en forme de manifeste ou peut-être plus justement d'explication des raisons de l'existence de cette association : quelle est le sort fait à l'économie dans la réflexion de l'APAD? Même si elle est extraite d'un ouvrage qui est un peu ancien (1985), appartenant d'une certaine manière à la "préhistoire de l'APAD" (bulletin de l'APAD nº 1 ), cette définition de J.-P. Olivier de Sardan pose clairement les termes de mon interrogation : "Nous entendrons par sciences sociales l'ensemble "ethnologie-sociologie", c'est à dire l'étude multidimensionnelle des groupes sociaux de toute nature, de leurs pratiques et de leurs représentations (l'approche historique étant ici conçue comme une composante interne à une telle étude, et qui lui est indispensable)" (p.7).

2 Le choix de ce passage peut paraitre anachronique, mais il me semble qu'il exprime bien une conception des sciences sociales que l'on retrouve sous une forme plus diffuse dans les textes fondateurs de l'APAD (J.-P. Olivier de Sardan, bulletin $n^{\circ} 1$ ).

Or une acception des sciences sociales réduite à l'ensemble ethnologie-sociologie, ensemble intégrant l'histoire comme composante interne et nécessaire Evans-Pritchard ouvrait la voie, lorsqu'il voyait entre historiographie et anthropologie sociale "des différences de technique, d'accentuation et de perspective et non des différences de méthode et de but" (1974, mais le texte date de 1950) -, ne dit rien de la place géographique réservée à l'économie, extérieure ou intérieure, et fonctionnelle, connexe ou subordonnée.

En lisant entre les lignes - en sur-interprétant ? -, on peut l'imaginer subordonnée, à l'instar de l'histoire, au binôme dominant, ou plutôt faux binôme: la distinction sociologie-anthropologie correspond à l'histoire de la structuration du monde universitaire, elle-même partiellement subordonnée à l'histoire économique de l'occident, marquée par la révolution industrielle, la domination des villes sur les campagnes et la colonisation, plutôt qu'à une nécessité scientifique. 
5 L'étude multidimensionnelle des pratiques des groupes sociaux ne peut se passer de celle des raisons de ces pratiques, donc de la rationalité qui guide les agents, rationalité économique pour partie (certes, cette vision de l'économie n'a pas grand chose à voir avec le reflet renvoyé par le miroir déformant de l'économétrie). L'économie apparaît alors comme un "angle d'attaque" pour la recherche en sciences sociales, dont la vocation est manifestement totalisante ("holiste"), même si elle doit échapper aux risques de substantification de concepts telles que" Société", "État", etc...

Point n'est besoin de remonter aux grands ancêtres Marx ou Weber, ou, sur un autre mode, à Karl Polanyi pour juger de la pertinence de cette idée. Il est en effet un domaine, objet actuellement de bien des sollicitudes, où elle s'exprime avec d'autant plus de force que ce domaine est en quelque sorte le cœur de la discipline économique : il s'agit du marché. Les apories auxquelles conduisent les présupposés de la théorie néo-classique des marchés ${ }^{1}$ sont depuis longtemps connues, et dénoncées par des courants de pensée allant jusqu'à F.A. Hayek et ses disciples néo-libéraux ${ }^{2}$.

Des études récentes, telles que celles conduites depuis 1987 par l'équipe de recherche IRAM-INRA-UNB ${ }^{3}$ sur le commerce frontalier, les politiques agricoles, et les espaces régionaux en Afrique de l'ouest, ainsi que des recherches menées aux département d'économie et sociologie rurale de la FSA-UNB ${ }^{4}$, montrent à quel point l'analyse des réseaux où s'imbriquent relations ethniques - dans le sens où elles sont vécues comme telles par les agents concernées -, de parenté et de clientèle est indispensable à la compréhension du fonctionnement des marchés céréaliers ouest-africain. L'intensité des relations commerciales nouées entre opérateurs définit des espaces transfrontaliers - les "périphéries nationales" de John Igué -, dont la réalité dépasse de loin celle de marchés nationaux encore en voie de construction.

8 Il serait possible d'aller plus dans ce sens en concevant le marché sous la forme d'un champ (P. Bourdieu, 1980, 1992), espace social polarisé, structuré à la fois par les positions respectives des différents agents et institutions qui y participent et par les stratégies poursuivies par ces agents, stratégies sous contraintes des positions objectivement occupées.

9 Cette façon de raisonner relationnellement permet de mettre en évidence les déterminants sociaux et économiques des agents engagés dans le jeu, et donc leurs rationalités respectives, dominées par la mise en valeur des différentes formes de capital dont ils sont dotés : on voit bien en particulier que le marché ne définit pas un espace compréhensible en termes purement économiques, et que l'on assiste à des transformations continuelles et réversibles du capital social (parentèle, ethnie) et culturel (connaissances linguistiques) en capital économique (capacité de financement, profit commercial).

10 Un moment essentiel dans le raisonnement en termes de champ est la définition de la position occupée par un champ déterminé par rapport au champ du pouvoir. Il serait riche d'enseignements de situer les stratégies individuelles (et familiales) des commerçants - différentes selon les catégories -, vis à vis des pouvoirs administratifs et politiques, et ceci aux différents échelons hiérarchiques et géographiques.

11 Le faible degré de formalisation et l'inefficacité des tentatives officielles de contrôle des marchés céréaliers masquent peut-être une très forte imbrication - et des codifications précises, même si elles sont qualifiées d'informelles - des relations sociales entre commerçants et représentants du pouvoir (appartenance à des réseaux communs, 
stratégies d'alliances ou clientélaires). C'est peut-être le "trop d'Etat" trop souvent lancé comme un anathème, dans un contexte socio-politique africain marqué par la "néo-patrimonialisation" de l'Etat (J. F. Médard, 1991) qui permet justement ce jeu complexe entre pratiques, codes sociaux et règles institutionnelles. Quelles que soient les implications en termes de jugements de valeur ou de choix politiques induites par le constat de cette situation, il parait impossible de faire l'économie de sa connaissance précise.

Ce cas particulier des marchés agricoles ouest-africains illustre bien, me semble-t-il, l'importance de réfléchir à la place que doit occuper l'économie dans le complexe "sciences sociales". Cette idée n'a bien sûr pas le mérite de l'originalité - il suffit de penser à la "nouvelle sociologie économique" américaine apparue dans les années 70 -, mais elle mérite sans doute une petite place dans les débats de l'APAD.

Cotonou, le 19 mai 1993

\section{NOTES}

1.Pour l'école néo-classique, le marché fonctionne de façon optimale en situation de concurrence parfaite, c'est à dire lorsque les conditions suivantes sont remplies : acteurs rationnels (il s'agit de la rationalité supposée universelle de l'homo œeconomicus), produits homogènes et interchangeables, libre entrée sur le marché, transparence de l'information.

2.Hayek et ses disciples rejettent comme irréalistes et même dangereuses à leurs yeux parce que susceptibles de justifier une intervention de l'État pour corriger les imperfections du marché réel - certaines hypothèses de base des néo-classiques relatives au fonctionnement des marchés en concurrence parfaite, en particulier celles concernant la multiplicité d'agents incapables d'influencer individuellement le marché, l'homogénéité des marchandises et surtout la notion d"'information complète" qualifiée par Hayek de "fâcheusement obscure" (Lagueux, 1989).

3.IRAM : Institut de recherches et d'application des méthodes de développement ; INRA : Institut nationale de la recherche agronomique ; UNB : Université nationale du Bénin.

4.FSA -UNB : Faculté des sciences agronomiques de l'Université nationale du Bénin.

\section{AUTEUR}

\section{PIERRE-YVES LE MEUR}

Agro-économiste, Faculté des sciences agronomiques, Cotonou, BENIN 